ARTICLE

\title{
Giant nonlinear optical activity in two-dimensional palladium diselenide
}

\author{
Juan $\mathrm{Yu}^{1,2,3,8}$, Xiaofei Kuang ${ }^{3,8}$, Junzi $\mathrm{Li}^{4,8}$, Jiahong Zhong ${ }^{1,2}$, Cheng Zeng ${ }^{1,2}$, Lingkai Cao ${ }^{1,2}$, Zongwen Liu (1) ${ }^{5}$, \\ Zhouxiaosong Zeng ${ }^{6}$, Ziyu Luo ${ }^{6}$, Tingchao He (1) ${ }^{4 凶}$, Anlian Pan (i) ${ }^{6 凶}$ \& Yanping Liu (10 ${ }^{1,2,7 凶}$
}

Nonlinear optical effects in layered two-dimensional transition metal chalcogenides have been extensively explored recently because of the promising prospect of the nonlinear optical effects for various optoelectronic applications. However, these materials possess sizable bandgaps ranging from visible to ultraviolet region, so the investigation of narrow-bandgap materials remains deficient. Here, we report our comprehensive study on the nonlinear optical processes in palladium diselenide ( $\left.\mathrm{PdSe}_{2}\right)$ that has a near-infrared bandgap. Interestingly, this material exhibits a unique thickness-dependent second harmonic generation feature, which is in contrast to other transition metal chalcogenides. Furthermore, the two-photon absorption coefficients of 1-3 layer $\mathrm{PdSe}_{2}\left(\beta \sim 4.16 \times 10^{5}, 2.58 \times 10^{5}\right.$, and $1.51 \times$ $10^{5} \mathrm{~cm} \mathrm{GW}^{-1}$ ) are larger by two and three orders of magnitude than that of the conventional two-dimensional materials, and giant modulation depths $\left(\alpha_{\mathrm{s}} \sim 32 \%, 27 \%\right.$, and $\left.24 \%\right)$ were obtained in 1-3 layer $\mathrm{PdSe}_{2}$. Such unique nonlinear optical characteristics make $\mathrm{PdSe}_{2}$ a potential candidate for technological innovations in nonlinear optoelectronic devices.

\footnotetext{
${ }^{1}$ School of Physics and Electronics, Hunan Key Laboratory for Super-microstructure and Ultrafast Process, Central South University, Changsha, Hunan, People's Republic of China. ${ }^{2}$ State Key Laboratory of High-Performance Complex Manufacturing, Central South University, Changsha, Hunan, People's Republic of China. ${ }^{3}$ School of Electronics and Information, Hangzhou Dianzi University, Zhejiang, People's Republic of China. ${ }^{4}$ Key Laboratory of Optoelectronic Devices and Systems of Ministry of Education and Guangdong Province, College of Physics and Optoelectronic Engineering, Shenzhen University, Shenzhen 518060, People's Republic of China. ${ }^{5}$ School of Chemical and Biomolecular Engineering, The University of Sydney, Sydney, NSW, 2006 , Australia. ${ }^{6}$ College of Materials Science and Engineering, Hunan University, Changsha, Hunan, People's Republic of China. ${ }^{7}$ Shenzhen Research Institute of Central South University, Shenzhen, People's Republic of China. ${ }^{8}$ These authors contributed equally: Juan Yu, Xiaofei Kuang, Junzi Li. ${ }^{凶}$ email: tche@szu.edu. cn; anlian.pan@hnu.edu.cn; liuyanping@csu.edu.cn
} 
$\mathrm{N}$ onlinear optical (NLO) properties play an increasingly significant role in the development of laser technology, optical spectroscopy, and material structure analysis methodologies ${ }^{1-4}$. Recently, the study of NLO activity in twodimensional (2D) layered materials (such as graphene, transition metal chalcogenides (TMDCs), and black phosphorus (BP)) has made significant progress ${ }^{5-12}$. The extraordinary nonlinearities of graphene, including saturable absorption (SA) ${ }^{13,14}$, optical limiting ${ }^{15}$ and harmonic generation ${ }^{16}$, have attracted great interest. However, the inherent properties of graphene, like its zerobandgap and centrosymmetric crystal structure ${ }^{17}$, hinder the study of its NLO effects, including the second harmonic generation (SHG) and multiphoton absorption features. On the contrary, BP possesses intrinsic anisotropy, strong light-matter interaction, layer-dependent direct bandgap $(0.3-2 \mathrm{eV})$, and a wide range of adjustable optical response ${ }^{18,19}$, leading to high SA modulation depths $\left(\sim 27.6 \%\right.$ at $400 \mathrm{~nm}, \sim 12.4 \%$ at $\left.800 \mathrm{~nm}^{20}\right)$ and a large two photon absorption (TPA) coefficient $((-6.98 \pm 0.6) \times$ $10^{3} \mathrm{~cm} \mathrm{GW}^{-121}$. However, BP lacks sufficient air stability, resulting in the rapid degradation of its NLO properties. Meanwhile, the most studied TMDC materials $\left(\mathrm{MX}_{2}, \mathrm{M}=\mathrm{Mo}, \mathrm{W}\right.$, and $\mathrm{X}=\mathrm{S}, \mathrm{Se}, \mathrm{Te}$ ) that exhibit various fascinating NLO activities, such as SHG, SA and TPA, have sizable bandgaps ranging from visible to the ultraviolet region $(1-2.5 \mathrm{eV})$ and there is a shortage of narrow-bandgap materials ${ }^{22}$. Interestingly, $\mathrm{PdSe}_{2}$ is a pentagonal 2D material with robust anisotropy, high carrier mobility, and air stability, that give it considerable advantages in nonlinear optoelectronic devices ${ }^{23}$. In addition, $\mathrm{PdSe}_{2}$ has a modifiable thicknessdependent bandgap from 0.03 to $1.37 \mathrm{eV}^{24}$, filling the interspace between zero-gap graphene and large-gap TMDCs. Meanwhile, $\mathrm{Xu}$ et al. demonstrated the ultrafast SA performance of liquidphase exfoliated $\mathrm{PdSe}_{2}$ that generated the dissipative soliton and pulse energ $y^{25}$. Zhang et al. experimentally investigated the modelocked $\mathrm{Er}$ and $\mathrm{Yb}$-doped operations in multilayer $\mathrm{PdSe}_{2}{ }^{26}$. These works demonstrate the capacity of $\mathrm{PdSe}_{2}$ for broadband ultrafast photonic devices. However, to date, the current experimental investigation of the NLO activities of $\mathrm{PdSe}_{2}$ (especially the monolayer sample) is insufficient. Therefore, an extensive and indepth study of the NLO behavior of $\mathrm{PdSe}_{2}$ is of great significance for the expansion of its optoelectronic applications.

Herein, we report the fabrication of large-size $\mathrm{PdSe}_{2}$ flakes with different layers and present a comprehensive investigation of thickness-dependent NLO properties of the $\mathrm{PdSe}_{2}$ flakes. Extraordinarily, owing to its unique layer-dependent inversion symmetry, $\mathrm{PdSe}_{2}$ exhibits a SHG behavior that is totally opposed to that in other $2 \mathrm{D}$ materials ${ }^{27,28}$. Moreover, the $\mathrm{PdSe}_{2}$ flakes exhibit giant TPA coefficients $(\beta)$ that are two to three orders of magnitude larger compared to that of the conventional semiconductors ${ }^{29-31}$. Importantly, excellent saturable absorption was observed in 1-3 layer (L) PdSe 2 , with much higher modulation depths $\left(\alpha_{\mathrm{s}}\right)$ than that of the most other $2 \mathrm{D}$ materials ${ }^{32-34}$. These findings not only provide profound insights into the NLO features of this innovative nanomaterial, but also open an avenue for future nonlinear optoelectronic applications.

\section{Results}

Crystal structure and characterization of few-layer $\mathrm{PdSe}_{2}$ flakes. The unique optical characteristics of $\mathrm{PdSe}_{2}$ are correlated to its special structure. It has a stratiform structure that is identical to some of the other 2D materials (graphene, hexagonal boron nitride (hBN), BP, and TMDCs), as shown in Fig. 1a. The Pd and Se atoms combine to form a covalent bond within a monolayer, and the van der Waals (vdWs) force dominates the a

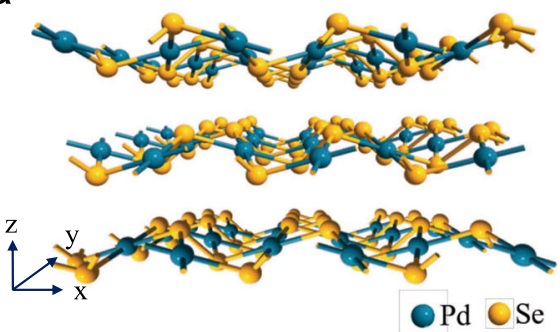

b

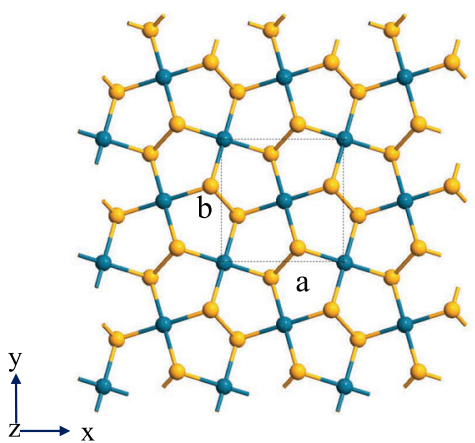

C

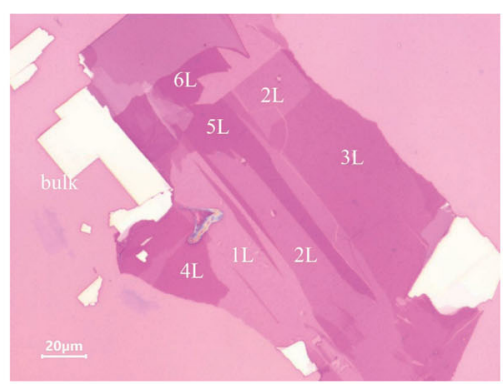

d

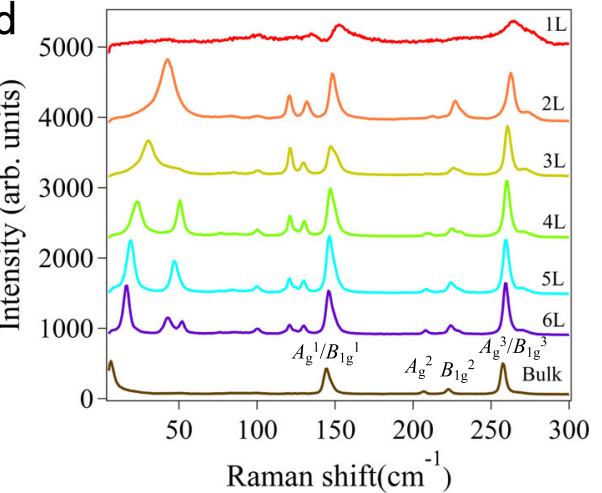

Fig. 1 Crystallographic structure and optical characteristics of a few-layer $\mathbf{P d S e}_{2}$. a The lattice structure of PdSe $e_{2}$ flakes with a puckered pentagonal. The unit cell vectors are denoted by the $x, y$, and $z$. Blue and yellow globes denote the Pd and Se atoms, respectively. $\mathbf{b}$ Top view of the atomic crystal structure of monolayer $\mathrm{PdSe}_{2}$. The black dotted frame represents a unit cell of $\mathrm{PdSe}_{2}$. The $a$ and $b$ denote the lattice constants along the $\mathrm{x}$ and $\mathrm{y}$ directions, respectively. c The optical microscope image of a few-layer $\mathrm{PdSe}_{2}$ on the silicon substrate with a $285 \mathrm{~nm} \mathrm{SiO}_{2}$ layer. The numbers of sample layers containing 1-6 L were marked with white letters, and the scale bar is $20 \mu \mathrm{m}$. d Raman spectra of the 1-6 L and bulk PdSe $\mathrm{flakes}_{2}$ with the same intensity axis. The low-frequency Raman peaks $\left(0-100 \mathrm{~cm}^{-1}\right)$ were robust and even exceeded that of the high-frequency modes. The Raman vibration modes of bulk $\mathrm{PdSe}_{2}$ were marked as $A_{\mathrm{g}}{ }^{1}, A_{\mathrm{g}}{ }^{2}, A_{\mathrm{g}}{ }^{3}, B_{1 \mathrm{~g}}{ }^{1}, B_{1 \mathrm{~g}}{ }^{2}$, and $B_{1 \mathrm{~g}}{ }^{3}$ mode. 
a

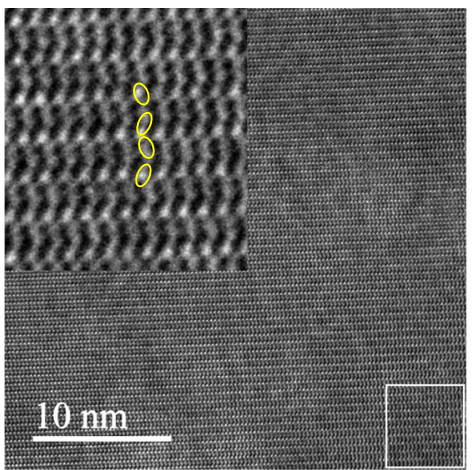

C

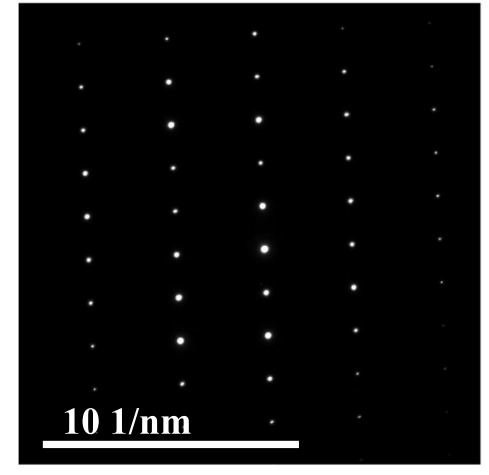

b
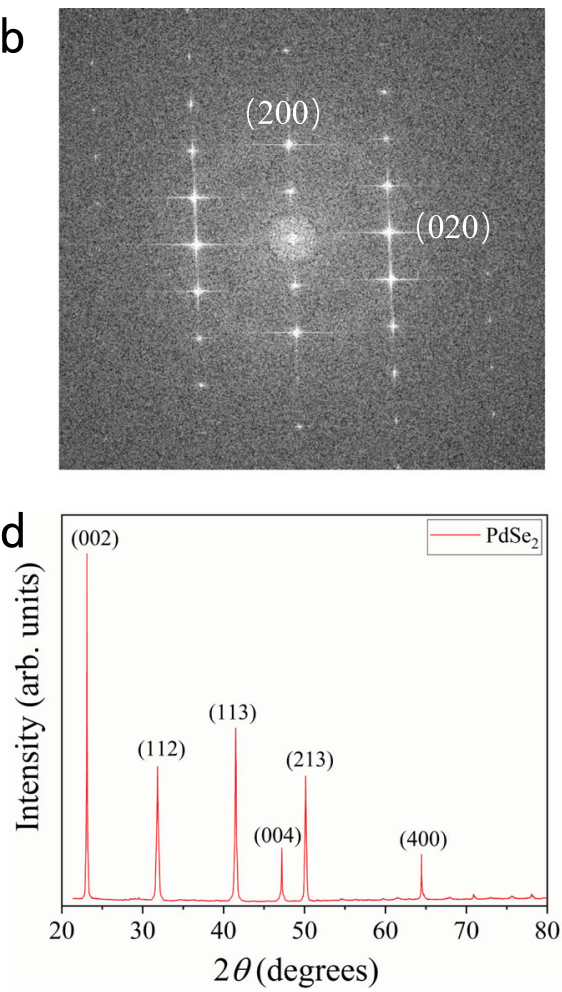

Fig. 2 TEM and XRD characterizations of $\mathbf{P d S e}_{\mathbf{2}}$ flakes. a HRTEM image of the few-layer region of PdSe $\mathrm{P}_{2}$ flakes. The inset shows a zoom-in of the white square regions, revealing the atomic models. The scale is marked in the lower-left corner. $\mathbf{b}$ FFT diffraction patterns of the solid white areas highlighted on the image (a). c SAED pattern of the PdSe 2 . d XRD pattern of bulk PdSe $e_{2}$ is in good agreement with the standard PDF card (PDF\#97-017-0327). The diffraction pattern exhibits six robust peaks at $23.1,31.8,41.5,47.2,50.14$, and $64.4^{\circ}$, respectively.

interactions between adjacent layers. In the side view, $\mathrm{PdSe}_{2}$ exhibits a unique plicate crystal structure with a monolayer vertical plicate height of $1.604 \mathrm{~A}$. In comparison to the traditional 2D materials with the highly symmetric hexagonal honeycomb structure, the $\mathrm{Pd}$ and $\mathrm{Se}$ atoms in $\mathrm{PdSe}_{2}$ constitute an asymmetric pentagonal structure (Fig. 1b). The ultrathin $\mathrm{PdSe}_{2}$ crystal displays robust interlayer coupling with a relatively large binding energy of $190 \mathrm{meV} /$ atom $^{35}$, as manifested in the process of mechanical exfoliation. Monolayer and few-layer $\mathrm{PdSe}_{2}$ flakes are difficult to be obtained by the conventional scotch tape $\operatorname{method}^{23,36}$. Instead, the gold-assisted exfoliation method was adopted to acquire the large-size monolayer and few-layer $\mathrm{PdSe}_{2}$, as shown in Fig. 1c on the $\mathrm{Si} / \mathrm{SiO}_{2}$ substrates. The height profiles of the $\mathrm{PdSe}_{2}$ samples were obtained by atomic force microscopy (AFM) measurements (Fig. S1 in Supplementary Information). According to the measurements, the average thickness of a single layer $\mathrm{PdSe}_{2}$ is $0.7 \mathrm{~nm}$. Recently, Raman technology has been demonstrated as an excellent approach in determining the layer number of various $2 \mathrm{D}$ materials ${ }^{37-40}$. Through analyzing the Raman spectrum, the layer number of a thin sample can be identified quickly, precisely and non-destructively. The Raman spectra of the 1-6 L and bulk $\mathrm{PdSe}_{2}$ samples at a wavenumber range of $0-300 \mathrm{~cm}^{-1}$ were obtained to estimate the layer numbers (Fig. 1d). Obviously, the Raman peak position varied gradually as the layer number increased. It is worth mentioning that the Raman peaks in the low-frequency region $\left(0-100 \mathrm{~cm}^{-1}\right)$ varied by a large margin. The low-frequency Raman modes indicate the occurrence of interlayer vibrations in the samples that were robust, and even exceeded those of the high-frequency modes $\left(100-300 \mathrm{~cm}^{-1}\right)$, which were attributed to the strong interlayer coupling of $\mathrm{PdSe}_{2}$. The peak positions of the low-frequency Raman modes have a specific relationship with the layer numbers, as shown in Fig. S2 in Supplementary Information. Because of the significant shifts of the low-frequency Raman modes, the layer number could be determined precisely. The feasibility and specific operation of such approach have been reported in detail in our previous work ${ }^{24}$.

Furthermore, the high-resolution transmission electron microscopy (HRTEM) characterization was performed to reveal the atomic structure of $\mathrm{PdSe}_{2}$. The periodic atomic arrangement of $\mathrm{PdSe}_{2}$ could be distinctly observed, and the sample had a flat surface (Fig. 2a). No obvious defects were observed, indicating high crystallinity of the samples. Figure $2 \mathrm{~b}$ shows the fast Fourier transform (FFT) diffraction patterns. The distinct diffraction spots correspond to the two principle crystal planes of (200) and (020). The selected-area electron diffraction (SAED) pattern was taken from the few-layer region of $\mathrm{PdSe}_{2}$ (Fig. 2c), indicating the high-quality single-crystal structure and confirming the isometric system of $\mathrm{PdSe}_{2}$. For the further investigation of our experimentally prepared $\mathrm{PdSe}_{2}, \mathrm{X}$-ray diffraction (XRD) characterization was performed. As shown in Fig. 2d, the diffraction pattern exhibits six robust peaks at $23.1,31.8,41.5,47.2,50.14$, and $64.4^{\circ}$, corresponding to (002), (112), (113), (004), (213), and (400) atomic planes of $\mathrm{PdSe}_{2}$, demonstrateing the high crystal quality of the sample ${ }^{41}$.

SHG properties of $\mathbf{P d S e}_{2}$ flakes. To better understand the NLO characteristics of the $\mathrm{PdSe}_{2}$ flakes, the SHG properties of fewlayer $\mathrm{PdSe}_{2}$ at the excitation wavelength of $800 \mathrm{~nm}$ were studied in detail. For the monolayer region, the detected SHG signal was negligible. When the laser spot was switched to the bilayer area, strong signals could be observed at $400 \mathrm{~nm}$. In order to further demonstrate the SHG signal dependence on the $\mathrm{PdSe}_{2}$ layer 
a

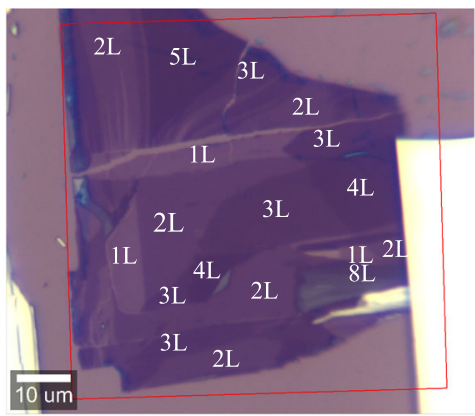

C

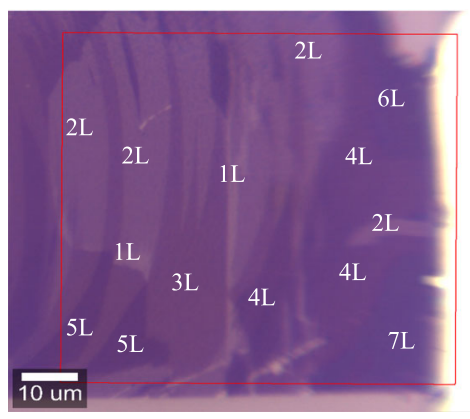

b

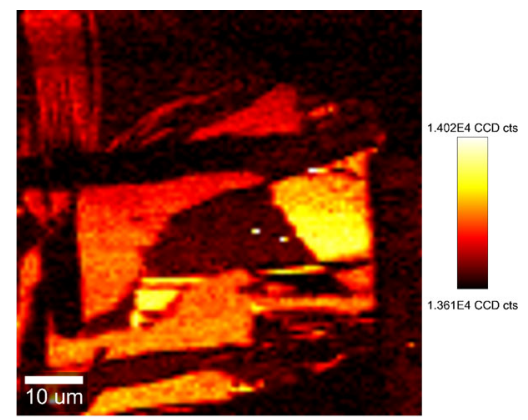

d

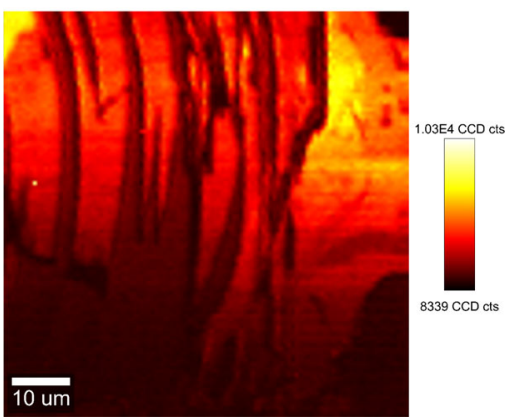

Fig. 3 SHG spatial mapping of few-layer $\mathbf{P d S e}_{2}$. $\mathbf{a}, \mathbf{c}$ The optical images of the PdSe $\mathrm{flakes}_{2}$ for the SHG characterization. The red boxes indicate the selected area for the SHG measurements. The layer numbers are labeled by the white numbers. The region elected in a contain $1-5 L$ and $8 L$ PdSe 2 and the area selected in $\mathbf{c}$ includes $1-7 \mathrm{~L} \mathrm{PdSe}{ }_{2}$, ensuring sufficient sampling. The PdSe $\mathrm{P}_{2}$ samples were excited by 800 nm wavelength using $50 \times$ objective with a NA value of 0.55 . b, d The spatial mapping of the integrated SHG signals corresponding to the regions in a and $\mathbf{c}$, respectively. The intensities of the SHG signals are different for different layers. The SHG signal is almost absent in the odd-layer PdSe 2 , while it is strong in the even-layer PdSe $\mathrm{W}_{2}$ The color bars are shown on the right of the figure.

number, the spatial mapping was implemented. The red box in Fig. 3a indicates the scanning regions of the sample, containing 1-5 L, $8 \mathrm{~L}$, and bulk $\mathrm{PdSe}_{2}$ flakes. Clearly, the SHG signals from even-numbered layers of $\mathrm{PdSe}_{2}$ were strong, while the signals from odd-numbered layers and the bulk $\mathrm{PdSe}_{2}$ were almost negligible (Fig. 3b). In order to ensure sufficient sampling, spatial scanning of another sample (consisting of 1-7 L and bulk $\mathrm{PdSe}_{2}$ ) was carried out and the same results were obtained, as can be seen in Fig. 3c, d. Such characteristics are exactly opposite to the SHG phenomenon displayed by other group VI 2D materials, where a strong SHG signal was only observed from the odd-numbered layers, and no signal was detected from even-numbered layers $27,28,42$. It is well known that strong SHG signals will occur only in 2D materials with a broken inversion symmetry ${ }^{43}$. Interestingly, the odd-numbered layers of $\mathrm{PdSe}_{2}$ belong to the $C_{2 \mathrm{~h}}$ $(2 / m)$ point group and $P 2_{1} / c$ (No.14) space group, which have an inversion symmetry, while the even-numbered layers possess a $C_{2 \mathrm{v}}(\mathrm{mm} 2)$ point group and $\mathrm{Pca}_{1}$ (No. 29) space group with a broken inversion symmetry ${ }^{23}$. Meanwhile, the bulk $\mathrm{PdSe}_{2}$ has an orthogonal structure with a $D_{2 \mathrm{~h}}$ point group and $\mathrm{Pbca}$ (No. 61) space group with an inversion symmetry ${ }^{23}$. These characteristics, that lead to the unique layer dependence of the SHG signal of $\mathrm{PdSe}_{2}$ flakes, are very unlike that of the other $2 \mathrm{D}$ materials that have an inversion symmetry in the even layers and a broken inversion symmetry in the odd layers ${ }^{42,44}$. Additionally, it was found that the $\mathrm{PdSe}_{2}$ samples were very uniform in thickness, attributing to the gold-assisted exfoliation method.

Generally, there are many factors that can influence the SHG intensity of $\mathrm{PdSe}_{2}$, and among those the excitation wavelength is relatively a significant one. To determine the wavelength that could generate the highest SHG signal, the SHG spectra were acquired by changing the excitation wavelengths from 780 to $1000 \mathrm{~nm}$ at a step length of $20 \mathrm{~nm}$. As shown in Fig. 4a, there were strong excitation wavelength-dependent optical signals. The highest optical signal was observed under $880 \mathrm{~nm}$ excitation, which could be caused by the SHG effect. To explore the physical mechanism under excitation at $880 \mathrm{~nm}$, the correlation between the detected optical intensity and the excitation intensity was measured. As presented in Fig. 4b, it was found that the optical intensity was quadratically dependent on the excitation power, and this was definitely the SHG signal. In addition, when excited with the same wavelength, the layer-dependent SHG signals of $\mathrm{PdSe}_{2}$ were measured. As expected, the odd-numbered layers (1, 3,5 , and $7 \mathrm{~L}$ ) exhibited very low SHG intensities while the evennumbered layers $(2,4,6$, and $8 \mathrm{~L})$ displayed much stronger SHG signals. The SHG intensities as a function of the layer numbers were plotted in Fig. $4 \mathrm{c}$, and it can be seen that the highest SHG intensity occurred in $4 \mathrm{~L} \mathrm{PdSe} e_{2}$ (inset). The SHG intensity of $\mathrm{PdSe}_{2}$ is mainly affected by two factors, the asymmetry and absorption intensity ${ }^{28,45}$. The odd-numbered layers of $\mathrm{PdSe}_{2}(1$, 3,5 , and $7 \mathrm{~L}$ ) reserve the symmetry so that no distinct SHG signal is detected. However, as the layer number increased, the signal was weak and non-zero. This was due to the small but finite optical phase shift between the $\mathrm{PdSe}_{2}$ layers ${ }^{28}$. Additionally, in the even-numbered layers $(2,4,6$, and $8 \mathrm{~L})$ of $\mathrm{PdSe}_{2}$, as the layer number increased, the asymmetry of $\mathrm{PdSe}_{2}$ increased, which caused a strong SHG signal in $4 \mathrm{~L} \mathrm{PdSe}_{2}{ }^{46}$. However, when the layer number was more than $4 \mathrm{~L}$, the optical absorption intensity in $\mathrm{PdSe}_{2}$ became much stronger, leading to a lower collection efficiency of $\mathrm{SHG}^{47}$. Therefore, influenced by these two factors, the $4 \mathrm{~L} \mathrm{PdSe} e_{2}$ exhibited the strongest SHG intensity. In general, the NLO process in a crystal can be expressed by the relationship of polarization vector and the electric field of the incident light in the form of ${ }^{1,2}$ :

$$
\mathbf{P}=\varepsilon_{0}\left(\chi \mathbf{E}+\chi^{(2)} \mathbf{E}^{2}+\chi^{(3)} \mathbf{E}^{3}+\cdots\right)
$$

where $\mathbf{P}$ is the polarization vector, and $\varepsilon_{0}$ is the permittivity in a 
a

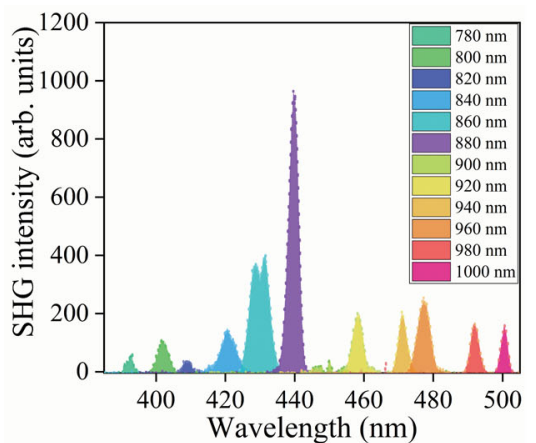

C

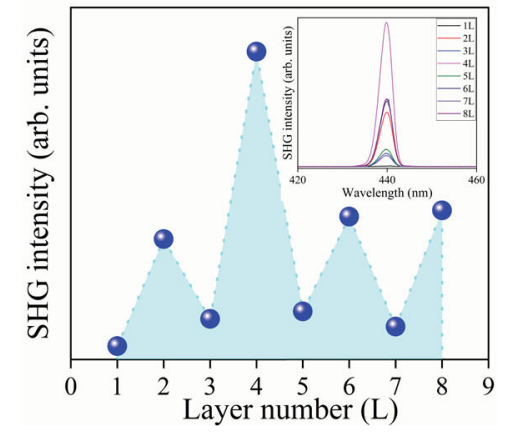

$b$

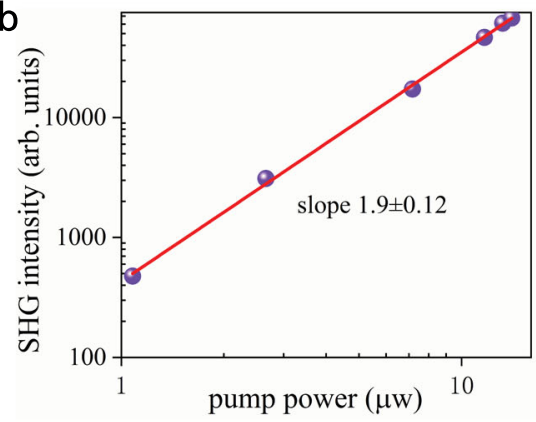

d

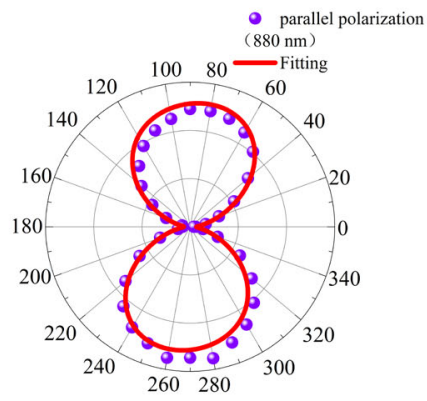

Fig. 4 SHG characterization of the $\mathbf{P d S e}_{\mathbf{2}}$ flakes. a The SHG intensities excited at various wavelengths from 780 to $1000 \mathrm{~nm}$, represented by different colors. The highest intensity appeared at the wavelength of $440 \mathrm{~nm}$, manifesting the strongest SHG signal of excitation at $880 \mathrm{~nm}$. $\mathbf{b}$ The excitation power dependence of $\mathrm{SHG}$ intensities in $4 \mathrm{LPdSe}$. Both axes are shown on the log scale. The correlation of the pump power and $\mathrm{SHG}_{\text {intensities exhibits was }}$ linear with a slope of $1.9 \pm 0.12$. c The SHG signal excited at the fixed wavelength of $880 \mathrm{~nm}$ in $1-8 \mathrm{~L} \mathrm{PdSe} \mathrm{P}_{2}$. The integrated intensity of SHG varied with different layers, represented by the blue dots. The SHG signal was the strongest in the $4 \mathrm{~L}$ sample. The inset represents the SHG intensities versus the layer numbers of $\mathrm{PdSe}_{2}$. d The purple dots indicate the polar plot of integrated SHG intensities excited by the $880 \mathrm{~nm}$ laser in $4 \mathrm{~L} \mathrm{PdSe} e_{2}$. The corresponding fitting result is denoted by the solid red curve.

vacuum, while $\chi$ is the electric susceptibility of the medium, and $\mathbf{E}$ is the electric field component of the incident light. For the SHG process, the dominated term is $\mathbf{E}^{2}$. The second-order electric susceptibility, $\chi^{(2)}$, reflects the SHG efficiency, and for $4 \mathrm{~L} \mathrm{PdSe}{ }_{2}$, it was determined to be $5.17 \times 10^{-11} \mathrm{~m} \mathrm{~V}^{-1}$ at the excitation of $880 \mathrm{~nm}$, according to Eqs. S1-3 in the Supplementary Information (Note S1). The $\chi^{(2)}$ values for different $2 \mathrm{D}$ materials are presented in Table S1. It can be seen that the $\chi^{(2)}$ value of $\mathrm{PdSe}_{2}$ is comparable to that of the other $2 \mathrm{D}$ materials.

Besides, the polarization-resolved SHG signals were also acquired, with the aim of identifying the crystal orientation of the sample. In our measurement, the polarization orientations of the incident and detected lights were parallel (for more specific information, see Experimental details). The experimental configuration and the measured parameters of the SHG measurement are illustrated in Fig. S3 in the Supplementary Information. Under a parallel configuration, the polar plot of the SHG signals represented distinct anisotropy, embodied in a dumbbell shape (Fig. 4d). The SHG intensity had its minimum at $0^{\circ}$, then it gradually increased as the polarization orientation rotated, reaching the maximum at $90^{\circ}$. The slight skewing of the polar plot might be resulted from the distorted crystal lattice. The nonlinear dielectric susceptibility tensor was employed to analyze the polarization-resolved SHG signals. The SHG polarization vector, $\mathbf{P}$, is related to the electrical field, $\mathbf{E}$, of the incident light, and their relationship is expressed as ${ }^{46}$

$$
\mathbf{P}=d \mathbf{E}
$$

where $d$ is the dielectric susceptibility tensor. Under the threedimensional geometric space, both $\mathbf{P}$ and $\mathbf{E}$ have three components in the $\mathrm{x}, \mathrm{y}$, and $\mathrm{z}$ directions. Likewise, other variables also have a three-dimensional form. Therefore, the above formula can be further expressed as follows

$$
\left(\begin{array}{c}
P_{x} \\
P_{y} \\
P_{z}
\end{array}\right)=\left(\begin{array}{llllll}
d_{11} & d_{12} & d_{13} & d_{14} & d_{15} & d_{16} \\
d_{21} & d_{22} & d_{23} & d_{24} & d_{25} & d_{26} \\
d_{31} & d_{32} & d_{33} & d_{34} & d_{35} & d_{36}
\end{array}\right)\left(\begin{array}{c}
E_{x} E_{x} \\
E_{y} E_{y} \\
E_{z} E_{z} \\
2 E_{y} E_{z} \\
2 E_{x} E_{z} \\
2 E_{x} E_{y}
\end{array}\right)
$$

where $d_{i j}$ is the element of the dielectric susceptibility tensor. For the experimental backscattering setup, the electrical field intensity of the incident light in the z-direction was negligible. Thus, the electrical field intensities in the $\mathrm{x}$ and $\mathrm{y}$ directions can be expressed as

$$
\begin{aligned}
& E_{x}=E_{0} \cos \theta \\
& E_{y}=E_{0} \sin \theta
\end{aligned}
$$

where $E_{0}$ is the module of the electrical field intensity of the incident light, and $\theta$ is the angle between the $x$-axis of the crystal lattice of $\mathrm{PdSe}_{2}$ and the polarization orientation of the incident light. Under the parallel backscattering configuration, the eventual SHG intensity is only correlated with $P_{x}$ and $P_{y}$, expressed as

$$
I_{\|} \propto\left(P_{x} \cos \theta+P_{y} \sin \theta\right)^{2}
$$

where $I_{\|}$is the detected SHG intensity under the parallel 
a

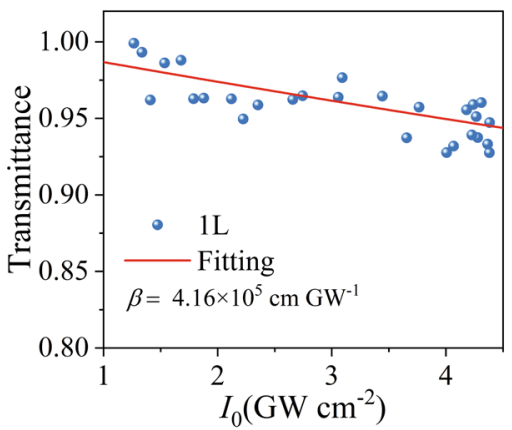

C

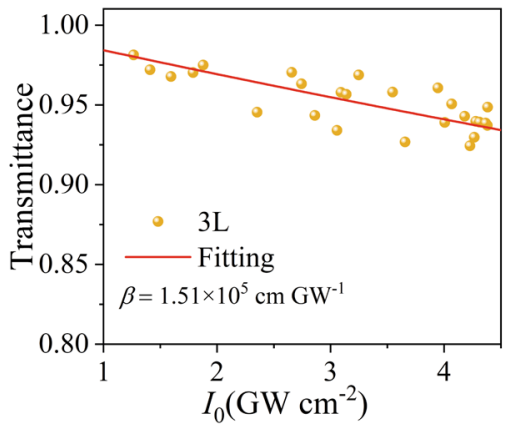

b

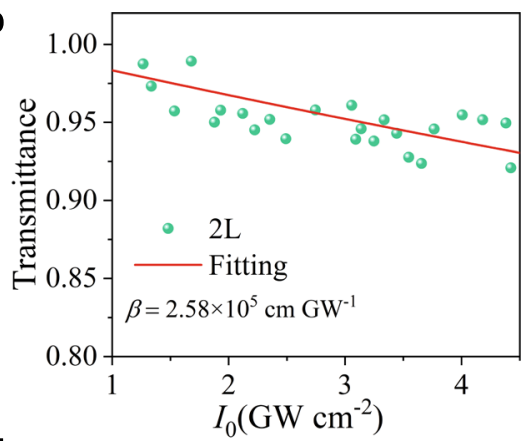

d

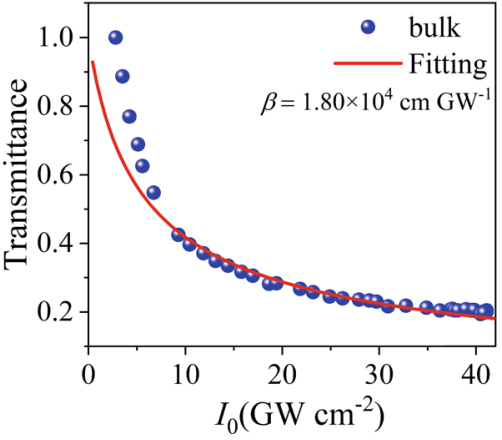

Fig. 5 TPA properties of 1-3 $\mathbf{L}$ and bulk PdSe $\mathbf{~}_{\mathbf{2}}$ flakes. a-c Nonlinear transmittance of 1-3 L PdSe $e_{2}$. The blue, green, and yellow dots represent the experimental data, while the solid red lines show the fitted results using the TPA model. The TPA coefficients $(\beta)$ of 1,2 , and $3 \mathrm{~L} \mathrm{PdSe} 2$ are $4.16 \times 10^{5}$, $2.58 \times 10^{5}$, and $1.51 \times 10^{5} \mathrm{~cm} \mathrm{GW}^{-1}$, respectively. $\mathbf{d}$ Nonlinear transmittance of bulk $\mathrm{PdSe}_{2}$. The blue dots represent the experimental results, while the solid red lines show the fitted results using the TPA model. The TPA coefficient $(\beta)$ of bulk PdSe 2 is $1.80 \times 10^{4} \mathrm{~cm} \mathrm{GW}^{-1}$. All the above measurements were performed under $800 \mathrm{~nm}$ excitation.

configuration. Substitute Eq. (3) with Eq. (5), we obtained

$$
I_{\|} \propto\left[\begin{array}{c}
d_{11} \cos \theta^{3}+\left(d_{21}+2 d_{16}\right) \cos \theta^{2} \sin \theta \\
+\left(d_{12}+2 d_{26}\right) \cos \theta \sin \theta^{2}+d_{22} \sin \theta^{3}
\end{array}\right]^{2}
$$

Using this formula, the experimental SHG intensities were fitted appropriately, as shown by the solid red curve in Fig. 4d. It can be seen that the experimental results are entirely consistent with the theoretical analysis. Meanwhile, the polarizationresolved SHG measurements are expected to accurately identify the crystal orientation of $\mathrm{PdSe}_{2}$ at the macro scale.

TPA properties of $\mathrm{PdSe}_{2}$ flakes. The NLO processes in 2D materials can be divided into two classifications, the parametric and non-parametric process ${ }^{48}$. The parametric process in $2 \mathrm{D}$ materials is mainly a scattering process, where the ground state is excited into a virtual state, and there is no electron transfer and absorption between the two virtual states, such as the SHG above. On the contrary, the non-parametric process is the excitation from the ground state to a real state, which involves absorption and electron transfer, mainly including TPA and SA. To deeply explore the NLO properties of $\mathrm{PdSe}_{2}$, its TPA was further investigated. Figure S4 illustrates the experimental setting of the home-built nonlinear transmittance system used to perform the TPA and SA measurements, together with the measured parameters. To perform the TPA measurement, the monolayer and few-layer $\mathrm{PdSe}_{2}$ flakes, obtained from the bulk crystal by goldassisted exfoliation, were transferred to the transparent quartz substrates. After that, their TPAs were measured using the nonlinear transmittance method. According to our previous reports, $\mathrm{PdSe}_{2}$ exhibits distinct linear dichroism features under the excitation of $300-800 \mathrm{~nm}^{24}$. When the excitation wavelength was 800 $\mathrm{nm}$, the linear absorption coefficients of 1,2 , and $3 \mathrm{~L} \mathrm{PdSe}_{2}$ were estimated to be $3.9 \times 10^{3}, 1.3 \times 10^{4}$ and $1.9 \times 10^{4} \mathrm{~cm}^{-1}$, respectively, according to the linear absorption measurement in Fig. S5 in Supplementary Information. Thus, the linear absorption was almost negligible in the solid film. Consequently, TPA could be predicted when excited by pulses at $800 \mathrm{~nm}$. As intuitively described in Fig. 5, the transmittance of the $\mathrm{PdSe}_{2}$ flakes decreased with the increasing of the incident light intensity, exhibiting a typical TPA characteristic.

Based on the nonlinear transmittance method, the TPA coefficients of the samples could be determined by the following formula ${ }^{49}$ :

$$
T\left(I_{0}\right)=\frac{I_{\mathrm{t}}}{I_{0}}=\frac{\left[\ln \left(1+I_{0} t \beta\right)\right]}{I_{0} t \beta}
$$

where $T\left(I_{0}\right)$ is the transmittance of the $\mathrm{PdSe}_{2}$ sample, and $I_{\mathrm{t}}$ is the transmitted light intensity of the sample, while $I_{0}$ is the incident irradiance intensity and $\beta$ is the TPA coefficient with $t$ being the thickness of the $\mathrm{PdSe}_{2}$ sample. By fitting the experimental data with Eq. (7), the $\beta$ values of $1,2,3 \mathrm{~L}$, and bulk $\mathrm{PdSe}_{2}$ were determined to be $4.16 \times 10^{5}, 2.58 \times 10^{5}, 1.51 \times 10^{5}$, and $1.80 \times 10^{4} \mathrm{~cm} \mathrm{GW}^{-1}$, respectively. These values are two to three orders of magnitude larger compared to those of other conventional semiconductors, such as $\mathrm{MoS}_{2}, \mathrm{WS}_{2}, \mathrm{GaAs}, \mathrm{CdS}$, and $\mathrm{ZnO}$ (Table S2 in Supplementary Information $)^{29-31,50}$, meaning the great potential of the few-layer PdSe $e_{2}$ flakes for TPA-based applications. Such giant TPA coefficients might originate from the strong exciton effects in $\mathrm{PdSe}_{2}{ }^{51}$. This was enhanced by the strong dielectric screening owing to the ultrathin structure of the monolayer ${ }^{23,52}$. In addition, it was found that the TPA coefficient of $\mathrm{PdSe}_{2}$ gradually decreased with the increasing of layers. Such a phenomenon was mainly attributed to the fact that the bandgap decreased with the increase of the number of layers, and there was a certain detuning between the formative exciton and the two-photon energy ${ }^{53}$.

SA properties of $\mathrm{PdSe}_{2}$ flakes. Considering that strong SA was widely reported in various two-dimensional TMDCs, including $\mathrm{MoS}_{2}{ }^{54}, \mathrm{WS}_{2}{ }^{55}, \mathrm{WSe}_{2}{ }^{56}$, and $\mathrm{MoSe}_{2}{ }^{57}$, a typical SA effect could 
a

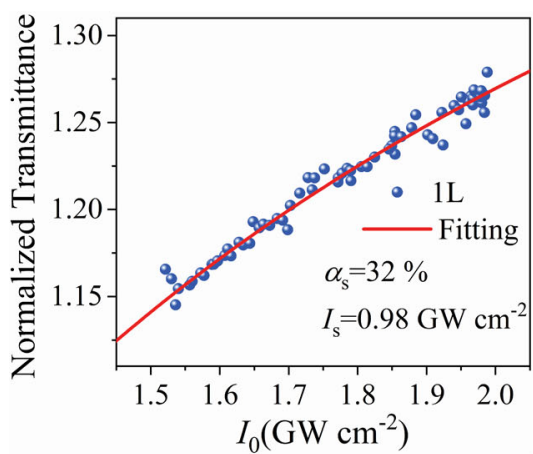

C

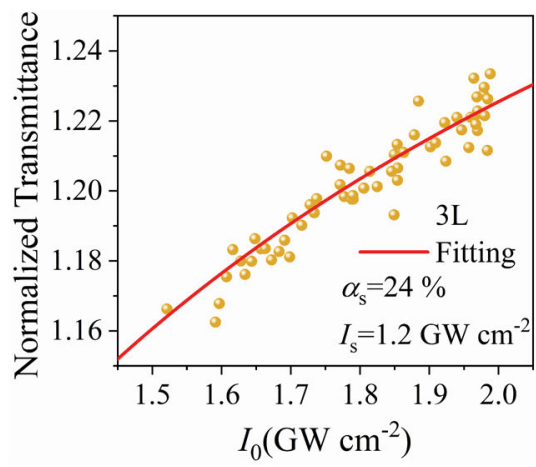

b

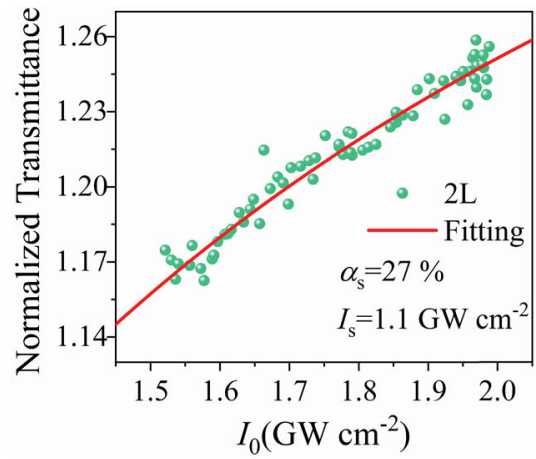

d

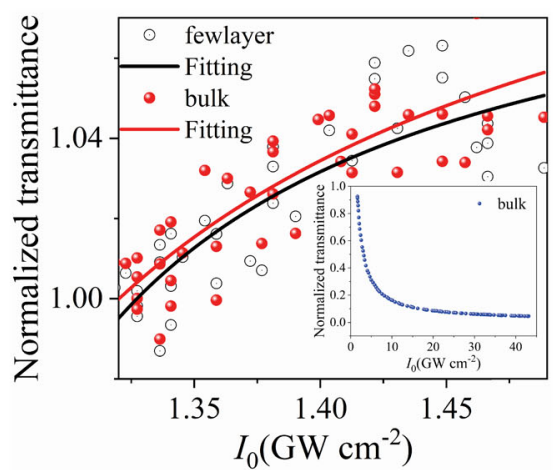

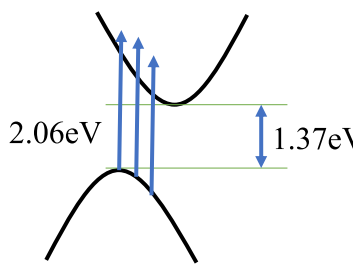
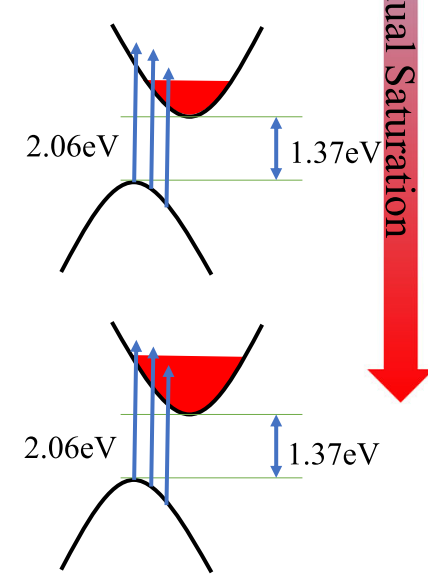

Fig. 6 SA properties of 1-3 $\mathbf{~}$ and bulk $\mathbf{P d S e}_{\mathbf{2}}$ flakes. a-c Nonlinear transmittance of 1-3 L PdSe ${ }_{2}$. The blue, green, and yellow dots represent the experimental data, while the solid red lines show the fitted results using the SA model. The SA intensities of 1,2 , and $3 \mathrm{~L} \mathrm{PdSe} 2$ are $0.98,1.1$, and $1.2 \mathrm{GW} \mathrm{cm}-2$, respectively. The modulation depths of 1, 2, and $3 \mathrm{~L} \mathrm{PdSe} 2$ are $32 \%, 27 \%$, and 24\%, respectively. d Nonlinear transmittance of the bulk PdSe 2 . The white and red dots represent the experimental data, while the black and red solid lines show the fitting curves of few-layer and bulk PdSe 2 using the SA model. The inset shows a reverse $\mathrm{SA}$ trend of the thick bulk PdSe 2 with increased laser power. e The electronic transition process of SA. All the above measurements were performed under $600 \mathrm{~nm}$ excitation.

also be expected in our $\mathrm{PdSe}_{2}$ in the case of $600 \mathrm{~nm}$ excitation. Figure 6 shows the trends of the normalized transmittance versus the incident intensity in 1-3 $\mathrm{L}$ and bulk $\mathrm{PdSe}_{2}$. The fitting could be performed according to a standard model of SA, as shown in the following formula ${ }^{58}$ :

$$
T\left(I_{0}\right)=1-\frac{\alpha_{\mathrm{s}}}{1+\frac{I_{0}}{I_{\mathrm{s}}}}-\alpha_{\mathrm{u}}
$$

where $\alpha_{\mathrm{s}}$ is the modulation depth, and $I_{\mathrm{s}}$ is the saturable intensity, while $\alpha_{\mathrm{u}}$ is the unsaturated absorption component. The obtained fitting parameters of the modulation depths $\left(\alpha_{\mathrm{s}}\right)$ of $1-3 \mathrm{~L} \mathrm{PdSe}_{2}$ were $32 \%, 27 \%$ and $24 \%$, respectively. The saturable intensities (usually the half of the highest optical absorption) of 1-3 $\mathrm{L} \mathrm{PdSe}_{2}$ were $0.98,1.1$, and $1.2 \mathrm{GW} \mathrm{cm}^{-2}$, respectively. As the sample thickened, the saturable intensity gradually increased. The modulation depths are much higher than that of the most other 2D materials, such as $\mathrm{SnSe}^{32}, \mathrm{MoSe}_{2}{ }^{33}, \mathrm{MoS}_{2}{ }^{34}$, and $\mathrm{PtSe}_{2}{ }^{59}$, which are promising for the application in ultrafast lasers. The high modulation depths mainly resulted from the low unsaturated loss, indicating a low defect density in the $\mathrm{PdSe}_{2}{ }^{7}$. However, the modulation depths in (thinner) bulk and few-layer $\mathrm{PdSe}_{2}$ (more than $10 \mathrm{~L}$, only $2.8 \%$ ) were much lower than that in $1-3 \mathrm{~L} \mathrm{PdSe} e_{2}$, which might be attributed to the increased unsaturated loss induced by the strengthened scattering ${ }^{7}$. It is worth noting that there was no SA phenomenon for the thicker bulk $\mathrm{PdSe}_{2}$ since when the sample was thick enough, the light capacity was sufficient, and the saturation state would not be easily reached. The excellent SA property of $1-3 \mathrm{~L} \mathrm{PdSe}_{2}$ endows its application prospect in the field of ultrafast lasers.

To understand the principle of the transmittance dependence on the incident irradiance, the electronic transition process in the momentum space should be clear. Figure 6e illustrates the electronic transition process of $\mathrm{SA}$ in $\mathrm{PdSe}_{2}$. The bandgap of the monolayer (bulk) $\mathrm{PdSe}_{2}$ was determined to be $1.37(0.03) \mathrm{eV}$ (Fig. S6 in Supplementary Information), indicating the unusual indirect bandgap characteristics. In our case, the energy of the incident photon $(2.06 \mathrm{eV})$ was much higher than the bandgap of a few-layer $\mathrm{PdSe}_{2}$. For low excitation intensity, the electrons in the valence band jumped to the relatively high energy levels in the conduction band, as illustrated on the top panel of Fig. 6e. Then, the hot electrons relaxed to the lower energy band, and populated in the bottom of the conduction band. With the increase in incident optical intensity, the number of excited electrons also increased. Electron is a fermion, that cannot populate at the same energy level. Thus, the energy levels were gradually filled from the bottom to the top in the conduction band (middle panel in Fig. 6e). When the energy level of the conduction band was filled with electrons as the incident light intensity increased, the electronic transition process was impeded owing to the Pauli exclusion principle. The number of the absorbed photons then gradually approached saturation, as indicated on the bottom panel of Fig. 6e. This explains the gradually saturable transmittance when the incident optical intensity was progressively increased.

In summary, large-area $\mathrm{PdSe}_{2}$ flakes with different thicknesses were successfully fabricated on the $\mathrm{Si} / \mathrm{SiO}_{2}$ and quartz substrates. Significantly, in contrast to that of the other 2D materials, robust broadband SHG properties in the even-numbered layers of $\mathrm{PdSe}_{2}$ were demonstrated, as a result of the unique anisotropic puckered pentagonal structure of $\mathrm{PdSe}_{2}$. Besides, the TPA and SA characteristics of the $\mathrm{PdSe}_{2}$ flakes were investigated. Under the excitation at $800 \mathrm{~nm}, 1-3 \mathrm{~L}$ and bulk $\mathrm{PdSe}_{2}$ exhibited giant TPA coefficients $(\beta)$ of $4.16 \times 10^{5}, 2.58 \times 10^{5}, 1.51 \times 10^{5}$, and $1.80 \times$ $10^{4} \mathrm{~cm} \mathrm{GW}^{-1}$, respectively, which are at least two orders of 
magnitude larger than those of the conventional semiconductors. Interestingly, when the excitation was switched to $600 \mathrm{~nm}$, on account of the ultra-low unsaturable loss, strong SA with the modulation depths $\left(\alpha_{\mathrm{s}}\right)$ up to $32 \%, 27 \%$ and $24 \%$ for $1-3 \mathrm{~L} \mathrm{PdSe}_{2}$ were observed. Compared to those of the other traditional 2D materials, these values are exceptionally large. Such prominent $\mathrm{NLO}$ activity of $2 \mathrm{D} \mathrm{PdSe} \mathrm{P}_{2}$ provides great prospects for potential applications in optical switching, ultrafast lasers, saturation absorbers, optical limiters, and micro/nano optical modulation devices.

\section{Methods}

Sample preparation. The $\mathrm{PdSe}_{2}$ flakes were exfoliated from bulk single-crystal $\mathrm{PdSe}_{2}$ (HQ graphene) onto silicon and transparent quartz substrates, employing the gold-assisted exfoliation. The thicknesses of the 1, 2, $3 \mathrm{~L}$, and bulk $\mathrm{PdSe}_{2}$ were $0.7,1.4,2.1$, and $10.5 \mathrm{~nm}$, respectively.

Raman single-spectrum characterization. A WITec Alpha $300 \mathrm{R}$ was utilized to obtain the high-resolution spectra. A $532 \mathrm{~nm}$ laser was used to excite the $\mathrm{PdSe}_{2}$ flakes with a power of $0.25 \mathrm{~mW}$, a $100 \times$ objective with $\mathrm{NA}=0.9$, and a laser spot size of $1 \mu \mathrm{m}$. The integration time was $5 \mathrm{~s}$ with accumulated ten times to obtain a high signal-to-noise ratio. The $1800 \mathrm{~g} \mathrm{~mm}^{-1}$ optical grating was used to improve the spectral resolution.

SHG measurements and characterization. The reflective geometry with normal incidence excitation was used to achieve the SHG measurements. The ultrashort 80 fs pulses with a $80 \mathrm{MHz}$ repetition rate (Spectra-Physics Solstice Maitai HP) were used as the excitation source for the SHG measurements. For SHG mapping characterization, the regions of $40 \times 40 \mu \mathrm{m}^{2}$ and $50 \times 50 \mu \mathrm{m}^{2}$ containing $1-8 \mathrm{~L}$ $\mathrm{PdSe}_{2}$ flakes were selected. A fiber-based pulsed laser was used as the fundamental pump source with the center wavelength at $800 \mathrm{~nm}$. The laser pulses were focused onto a spot size of $6 \mu \mathrm{m}$ on the $\mathrm{PdSe}_{2}$ sample by a $50 \times$ objective with $\mathrm{NA}=0.55$. The same objective lens was used to collect the SHG signal scattered from the sample and then detected by the charged coupling device (CCD) spectrometer. The integration time of $0.5 \mathrm{~s}$ and the scan duration of $2 \mathrm{~h}$ was set to achieve the highresolution mapping images.

For the measurements of layer number dependent SHG signal, the excitation wavelength was fixed at $880 \mathrm{~nm}$, and the same excitation intensity was used. For SHG polarization measurements, a half-wave plate (analyzer) was placed in the incident (outgoing) light path. Only the light with its polarization orientation parallel to the optical axis of the analyzer could pass and be detected by the CCD spectrometer. A step size of $10^{\circ}$ was adopted for the parallel configuration.

TPA and SA measurements. The TPA and SA of $\mathrm{PdSe}_{2}$ were measured using the nonlinear transmittance method in a home-built micro NLO system. The samples were excited at the wavelength of $600 \mathrm{~nm}$ (spectral width $\sim 15 \mathrm{~nm}$ ) or $800 \mathrm{~nm}$ (spectral width $\sim 10 \mathrm{~nm}$ ) by the laser pulses from an OPA combined with TOPAS ( $1000 \mathrm{~Hz}, 110 \mathrm{fs}$, Spectra-Physics, Inc.). The fs pulses were focused onto the sample by $\mathrm{A} 50 \times$ objective lens with $\mathrm{NA}=0.55$.

\section{Data availability}

All data generated during this study are included in this published article and its supplementary information files. All other relevant data are available from the corresponding authors on request.

Received: 24 June 2020; Accepted: 15 January 2021; Published online: 17 February 2021

\section{References}

1. Levenson, M. The principles of nonlinear optics. IEEE J. Quantum Electron. 21, 400-400 (1985).

2. Barry, R. M. \& Robert, W. B. Book review: nonlinear optics, 3rd edn. J. Biomed. Opt. 14, 1-4 (2009).

3. Shen, Y. R. Surface properties probed by second-harmonic and sum-frequency generation. Nature 337, 519-525 (1989).

4. Bloembergen, N. Nonlinear optics and spectroscopy. Rev. Mod. Phys. 54, 685-695 (1982).

5. Xia, F., Mueller, T., Lin, Y.-m, Valdes-Garcia, A. \& Avouris, P. Ultrafast graphene photodetector. Nat. Nanotechnol. 4, 839-843 (2009).

6. Ju, L. et al. Graphene plasmonics for tunable terahertz metamaterials. Nat. Nanotechnol. 6, 630-634 (2011).
7. Bao, Q. et al. Atomic-layer graphene as a saturable absorber for ultrafast pulsed lasers. Adv. Funct. Mater. 19, 3077-3083 (2009).

8. Tsai, D.-S. et al. Few-layer MoS2 with high broadband photogain and fast optical switching for use in harsh environments. ACS Nano 7, 3905-3911 (2013).

9. Liu, W. et al. Tungsten diselenide for mode-locked erbium-doped fiber lasers with short pulse duration. Nanotechnology 29, 174002 (2018).

10. Wang, H. \& Qian, X. Giant optical second harmonic generation in twodimensional multiferroics. Nano Lett. 17, 5027-5034 (2017).

11. Zhou, F. \& Ji, W. Giant three-photon absorption in monolayer MoS2 and its application in near-infrared photodetection. Laser Photon. Rev. 11, 1700021 (2017).

12. Wang, L. et al. Nonlinear optical signatures of the transition from semiconductor to semimetal in PtSe2. Laser Photon. Rev. 13, 1900052 (2019).

13. Sun, Z., Hasan, T. \& Ferrari, A. C. Ultrafast lasers mode-locked by nanotubes and graphene. Phys. E 44, 1082-1091 (2012).

14. Martinez, A. \& Sun, Z. Nanotube and graphene saturable absorbers for fibre lasers. Nat. Photon. 7, 842-845 (2013).

15. Wang, J. et al. Graphene and carbon nanotube polymer composites for laser protection. J. Inorg. Organomet. Polym. Mater. 21, 736-746 (2011).

16. Kumar, N. et al. Third harmonic generation in graphene and few-layer graphite films. Phys. Rev. B 87, 121406 (2013).

17. Nair, R. R. et al. Fine structure constant defines visual transparency of graphene. Science 320, 1308 (2008).

18. Tran, V., Soklaski, R., Liang, Y. \& Yang, L. Layer-controlled band gap and anisotropic excitons in few-layer black phosphorus. Phys. Rev. B 89, 235319 (2014).

19. Li, L. et al. Direct observation of the layer-dependent electronic structure in phosphorene. Nat. Nanotechnol. 12, 21-25 (2017).

20. Lu, S. B. et al. Broadband nonlinear optical response in multi-layer black phosphorus: an emerging infrared and mid-infrared optical material. Opt. Express 23, 11183-11194 (2015).

21. Chen, R., Tang, Y., Zheng, X. \& Jiang, T. Giant nonlinear absorption and excited carrier dynamics of black phosphorus few-layer nanosheets in broadband spectra. Appl. Opt. 55, 10307-10312 (2016).

22. Mak, K. F. \& Shan, J. Photonics and optoelectronics of 2D semiconductor transition metal dichalcogenides. Nat. Photon. 10, 216-226 (2016).

23. Oyedele, A. D. et al. PdSe2: Pentagonal two-dimensional layers with high air stability for electronics. J. Am. Chem. Soc. 139, 14090-14097 (2017).

24. Choi, Y. et al. Complete determination of the crystallographic orientation of $\mathrm{ReX} 2(\mathrm{X}=\mathrm{S}, \mathrm{Se})$ by polarized Raman spectroscopy. Nanoscale Horiz. $\mathbf{5}$, 308-315 (2020).

25. Huanian, Z. et al. Palladium selenide as a broadband saturable absorber for ultra-fast photonics. Nanophotonics 9, 2557-2567 (2020).

26. Nannan, X. et al. Palladium diselenide as a direct absorption saturable absorber for ultrafast mode-locked operations: from all anomalous dispersion to all normal dispersion. Nanophotonics 9, 4295-4306 (2020).

27. Malard, L. M., Alencar, T. V., Barboza, A. P. M., Mak, K. F. \& de Paula, A. M. Observation of intense second harmonic generation from MoS2 atomic crystals. Phys. Rev. B 87, 201401 (2013).

28. Li, Y. et al. Probing symmetry properties of few-layer MoS2 and h-BN by optical second-harmonic generation. Nano Lett. 13, 3329-3333 (2013).

29. Zhang, S. et al. Direct observation of degenerate two-photon absorption and its saturation in WS2 and MoS2 monolayer and few-layer films. ACS Nano 9, 7142-7150 (2015).

30. Li, Y. et al. Giant two-photon absorption in monolayer MoS2. Laser Photon. Rev. 9, 427-434 (2015).

31. Cirloganu, C. M., Padilha, L. A., Fishman, D. A., Webster, S., Hagan, D. J., \& Van Stryland, E. W. Extremely nondegenerate two-photon absorption in direct-gap semiconductors [Invited]. Opt. Express 19, 22951-22960 (2011).

32. Zhang, C. et al. Anisotropic nonlinear optical properties of a SnSe flake and a novel perspective for the application of all-optical switching. Adv. Optical Mater. 7, 1900631 (2019).

33. Luo, Z. et al. Nonlinear optical absorption of few-layer molybdenum diselenide $(\mathrm{MoSe} 2)$ for passively mode-locked soliton fiber laser [Invited]. Photon. Res. 3, A79-A86 (2015).

34. Luo, Z. et al. 1-, 1.5-, and 2- $\mu \mathrm{m}$ fiber lasers Q-switched by a broadband few-layer MoS2 saturable absorber. J. Lightwave Technol. 32, 4077-4084 (2014).

35. Yuan, H. et al. Polarization-sensitive broadband photodetector using a black phosphorus vertical p-n junction. Nat. Nanotechnol. 10, 707-713 (2015).

36. Liang, Q. et al. High-performance, room temperature, ultra-broadband photodetectors based on air-stable PdSe2. Adv. Mater. 31, 1807609 (2019).

37. O'Brien, M. et al. Mapping of low-frequency raman modes in CVD-grown transition metal dichalcogenides: layer number, stacking orientation and resonant effects. Sci. Rep. 6, 19476 (2016).

38. Ferrari, A. C. et al. Raman spectrum of graphene and graphene layers. Phys. Rev. Lett. 97, 187401 (2006). 
39. Yu, J. et al. Observation of double indirect interlayer exciton in WSe2/WS2 heterostructure. Opt. Express 28, 13260-13268 (2020).

40. Shi, W. et al. Raman and photoluminescence spectra of two-dimensional nanocrystallites of monolayer WS2 and WSe2. 2D Mater. 3, 025016 (2016)

41. Soulard, C. et al. Experimental and theoretical investigation on the relative stability of the PdS2- and pyrite-type structures of PdSe2. Inorg. Chem. 43, 1943-1949 (2004).

42. Ribeiro-Soares, J. et al. Second harmonic generation in WSe2. 2D Mater. 2, 045015 (2015).

43. Wang, Y., Xiao, J., Yang, S., Wang, Y. \& Zhang, X. Second harmonic generation spectroscopy on two-dimensional materials [Invited]. Opt. Mater. Express 9, 1136-1149 (2019).

44. Mennel, L., Paur, M. \& Mueller, T. Second harmonic generation in strained transition metal dichalcogenide monolayers: MoS2, MoSe2, WS2, and WSe2. APL Photon. 4, 034404 (2018).

45. Lin, X. et al. Two-dimensional pyramid-like WS2 layered structures for highly efficient edge second-harmonic generation. ACS Nano 12, 689-696 (2018).

46. Song, Y. et al. Extraordinary second harmonic generation in ReS2 atomic crystals. ACS Photon. 5, 3485-3491 (2018).

47. Song, Y. et al. Second harmonic generation in atomically thin MoTe2. Adv. Optical Mater. 6, 1701334 (2018)

48. Wen, X., Gong, Z. \& Li, D. Nonlinear optics of two-dimensional transition metal dichalcogenides. InfoMat 1, 317-337 (2019).

49. Qiu, X. et al. Strong multiphoton absorption in chiral CdSe/CdS dot/rod nanocrystal-doped poly(vinyl alcohol) films. Opt. Lett. 44, 2256-2259 (2019).

50. Liu, W. et al. Giant two-photon absorption and its saturation in 2D organic-inorganic perovskite. Adv. Optical Mater. 5, 1601045 (2017).

51. Van Stryland, E. W., Woodall, M. A., Vanherzeele, H. \& Soileau, M. J. Energy band-gap dependence of two-photon absorption. Opt. Lett. 10, 490-492 (1985).

52. Drüppel, M., Deilmann, T., Krüger, P. \& Rohlfing, M. Diversity of trion states and substrate effects in the optical properties of an MoS2 monolayer. Nat. Commun. 8, 2117 (2017).

53. Xie, Y. et al. Layer-modulated two-photon absorption in MoS2: probing the shift of the excitonic dark state and band-edge. Photon. Res. 7, 762-770 (2019).

54. Wang, K. et al. Ultrafast saturable absorption of two-dimensional MoS2 nanosheets. ACS Nano 7, 9260-9267 (2013).

55. Wei, C. et al. Passively Q-switched mid-infrared fluoride fiber laser around $3 \mu \mathrm{m}$ using a tungsten disulfide (WS2) saturable absorber. Laser Phys. Lett. 13, 105108 (2016).

56. Bikorimana, S. et al. Nonlinear optical responses in two-dimensional transition metal dichalcogenide multilayer: WS2, WSe2, MoS2 and Mo0.5W0.5S2. Opt. Express 24, 26998-26998 (2016).

57. Chen, B. et al. Q-switched fiber laser based on transition metal dichalcogenides MoS2, MoSe2, WS2, and WSe2. Opt. Express 23, 26723-26737 (2015).

58. Ren, C. et al. A near-infrared I emissive dye: toward the application of saturable absorber and multiphoton fluorescence microscopy in the deeptissue imaging window. Chem. Commun. 55, 5111-5114 (2019).

59. Yuan, J. et al. Few-layer platinum diselenide as a new saturable absorber for ultrafast fiber lasers. ACS Appl. Mater. Interfaces 10, 21534-21540 (2018).

\section{Acknowledgements}

The authors acknowledge support from the National Natural Science Foundation of China (Grant No. 61775241), the Youth Innovation Team (Grant No:2019012) of CSU, the Science and Technology Innovation Basic Research Project of Shenzhen (Grant No. JCYJ20180307151237242), Hunan Provincial Science Fund for Distinguished Young Scholars (Grant No: 2020JJ2059), and the Hunan province key research and development project (Grant No. 2019GK2233). The authors also are grateful for the support by the Project of State Key Laboratory of High-Performance Complex Manufacturing, Central South University (Grant No. ZZYJKT2020-12). Z.W.L. thanks the support from the Australian Research Council (ARC Discovery Project, DP180102976).

\section{Author contributions}

Y.P.L., A.L.P. and T.C.H. designed and managed the project. J.Y. fabricated the device and performed the Raman characterizations. J.Z.L., Z.X.S.Z. and Z.Y.L. performed the nonlinear optical measurements. Y.P.L., J.Y., X.F.K., C.Z., J.H.Z., L.C.C., Z.W.L. and T.C. H. provided a vital interpretation of the data. Y.P.L., T.C.H. and J.Y. drafted the paper All authors read and contributed to the revising of the manuscript. J.Y., X.F.K. and J.Z.L. contributed equally to this work.

\section{Competing interests}

The authors declare no competing interests.

\section{Additional information}

Supplementary information The online version contains supplementary material available at https://doi.org/10.1038/s41467-021-21267-4.

Correspondence and requests for materials should be addressed to T.H., A.P. or Y.L.

Peer review information Nature Communications thanks Shenggui Fu and the other, anonymous, reviewer(s) for their contribution to the peer review of this work.

Reprints and permission information is available at http://www.nature.com/reprints

Publisher's note Springer Nature remains neutral with regard to jurisdictional claims in published maps and institutional affiliations.

\begin{abstract}
Open Access This article is licensed under a Creative Commons Attribution 4.0 International License, which permits use, sharing,
adaptation, distribution and reproduction in any medium or format, as long as you give appropriate credit to the original author(s) and the source, provide a link to the Creative Commons license, and indicate if changes were made. The images or other third party material in this article are included in the article's Creative Commons license, unless indicated otherwise in a credit line to the material. If material is not included in the article's Creative Commons license and your intended use is not permitted by statutory regulation or exceeds the permitted use, you will need to obtain permission directly from the copyright holder. To view a copy of this license, visit http://creativecommons.org/ licenses/by/4.0/.
\end{abstract}

(C) The Author(s) 2021 